\title{
Caracterização de hastes de flores tropicais da emissão até a colheita ${ }^{(1)}$
}

\author{
VIVIAN LOGES ${ }^{(2)}$; ANA CECÍLIA R. DE CASTRO ${ }^{(3)}$; WALMA NOGUEIRA R. GUIMARÃES ${ }^{(4)}$; ANDREZA S. DA COSTA ${ }^{(3)}$; MARIA \\ DO CARMO FERRAZ TEIXEIRA ${ }^{(5)}$
}

\begin{abstract}
RESUMO
O desenvolvimento e o tempo para colheita de flores tropicais foram avaliados no Laboratório de Floricultura da Universidade Federal Rural de Pernambuco. Para tanto, foram mensuradas, separadamente, plantas das espécies Tapeinochilos ananassae, Zingiber spectabilis, Heliconia rostrata, H. rauliniana, Renanthera coccinea e variedades de Anthurium andraeanum, Alpinia purpurata e Etlingera elatior quanto ao comprimento e diâmetro das hastes e inflorescências desde o momento da emissão da inflorescência, em intervalos de 7 a 8 dias após a marcação (DAM) até o ponto de corte. Este experimento foi conduzido no município de Paulista (PE). As análises de variância foram efetuadas utilizando o Programa SAS. As médias dos tratamentos foram comparadas pelo teste de Tukey a 5\% de probabilidade. As hastes florais de A. purpurata variedades Vermelha, Jungle King e Jungle Queen atingiram o ponto de colheita aos 29, 37 e 43 DAM, respectivamente. As cultivares de A. andraeanum atingiram o ponto de corte entre 20 e 29 DAM. H. rauliniana e H. rostrata não apresentaram diferença para comprimento da inflorescência e da haste entre 9 e 15 DAM, atingindo, ambas espécies, o ponto de corte aos 15 DAM. As variedades de E. elatior atingiram o ponto de corte entre 36 e 42 DAM. Houve aumento do comprimento e diâmetro das inflorescências com o decorrer do tempo, porém o comprimento e diâmetro das hastes não diferiram no intervalo de 21 a 42 DAM. O Z. spectabilis atingiu o ponto de colheita entre os 29 e 37 DAM, porém como as inflorescências podem ser colhidas com tamanho maior, o ponto de corte ultrapassa os 42 DAM. As hastes florais de T. ananasseae a partir de 29 DAM atingiram o ponto de colheita e as hastes florais de R. coccinea aos 42 DAM. Os resultados indicaram que a data da colheita e a qualidade das inflorescências podem ser estimadas antecipadamente, a partir da emissão das inflorescências.
\end{abstract}

Palavras-chave: Zingiberaceae, Heliconiaceae, Araceae, Orchidaceae, ponto de corte.

\section{ABSTRACT}

\section{Tropical flowers stem characterization since the emergence to the harvest}

Development and time to harvest of some tropical flowers were evaluated at the Floriculture Laboratory of UFRPE. Tapeinochilos ananassae, Zingiber spectabilis, Heliconia rostrata, H. rauliniana, Renanthera coccinea and varieties of Anthurium andraeanum, Alpinia purpurata and Etlingera elatior where studied. On this experiment, length and diameter of both stem and inflorescence where measured from the moment of inflorescence emergence, at weekly intervals, up to cutting time. This experiment was conducted at a flower production farm in Paulista-PE. Analyses of variance were conducted with SAS, and means comparison by Tukey's test at the 5\% probability level. Stems of $A$. purpurata Red, Jungle King and Jungle Queen varieties, achieved harvest times at 29, 37 and 43 DAM, respectively. A. andraeanum cultivars could be cut between 20 and 29 DAM. H. rauliniana and $H$. rostrata did not increase inflorescence and stem length from 9 to 15 DAM, with cutting point at 15 DAM. E. elatior varieties attaining cutting point between 36 and 42 DAM. There was an increase in inflorescence length and diameter, but both stem length and stem diameter did not change from 21 to 42 DAM. Z. spectabilis achieved cutting point between 29 and 37 DAM but may be cut up to 42 DAM since bigger inflorescences are acceptable. T. ananassae stems achieved harvest condition from 29 DAM and flower stems of $R$. coccinea could be cut at 42 DAM. Our results indicate that harvest date and inflorescence quality may be estimated from inflorescence emission.

Key words: Zingiberaceae, Heliconiaceae, Araceae, Orchidaceae, harvest point.

\footnotetext{
${ }^{(1)}$ Apoio: Banco do Nordeste; Fazenda Mumbecas, Paulista (PE); UFRPE; CNPq; FACEPE; CAPES. Recebido em 07/04/2006 e aprovado para publicação em 11/02/2008.

${ }^{2)}$ Prof $^{\text {a }}$. Dra. Dep. Agronomia, Fitotecnia - UfRPE. Av. Dom Manuel de Medeiros, S/N, CEP: 52171-900, Recife (PE). e-mail: vloges@yahoo.com.

(3) Doutoranda PPGB-UFRPE: cecilia@cnpat.embrapa.br; costa.andreza@gmail.com.

(4) Bolsista FACEPE/PROMATA, Lab. de Floricultura-UFRPE: walmalamo@gmail.com.

(5) Fazenda Mumbecas, Paulista (PE) mariadocarmo@florestropicais.com.br.
} 


\section{INTRODUÇÃO}

A exportação de flores e plantas ornamentais do Brasil em 2006 foi da ordem de US\$29,63 milhões com um crescimento de 15,06\% em relação a 2005 (JUNQUEIRA e PEETZ, 2007). O Brasil apresenta vantagens para se especializar na produção de flores, devido aos microclimas, disponibilidade de terra, água, energia e mão-de-obra. Esse conjunto de fatores incide diretamente, na qualidade do produto e favorece custos mais baixos acarretando preços competitivos com os mercados externos.

As espécies tropicais ocorrem naturalmente na faixa tropical úmida da América, Ásia e Pacífico Oeste ou são exploradas em plantios convencionais (CASTRO, 1993). O desenvolvimento da floricultura tropical em Pernambuco foi significativo nos últimos anos em vários municípios da Zona da Mata de Pernambuco e em Petrolina, no sertão do estado. Na maioria das áreas produtoras são cultivadas espécies ou cultivares das famílias Zingiberaceae, Heliconiaceae, Araceae, Orchidaceae, além de vários tipos de folhagens (LOGES et al., 2005a).

Atualmente, o profissionalismo e competitividade do mercado de flores exigem elevados índices de produtividade, padronização e planejamento da produção, introdução de novas variedades, boa apresentação dos produtos e qualidade. Atentos a essas necessidades, os produtores estão organizados em associações e cooperativas, em busca de informações que possibilitem o crescimento da atividade segundo a exigência do mercado.

A falta de informação quanto ao desenvolvimento e tempo para colheita das principais espécies tropicais cultivadas, dificulta a organização dos produtores no momento da comercialização. Por isso, essa pesquisa tem como finalidade caracterizar o desenvolvimento de hastes florais de espécies tropicais do momento da emissão da inflorescência até a colheita.

\section{MATERIALEMÉTODOS}

$\mathrm{O}$ experimento foi conduzido no município de Paulista (07 $56^{\prime}$ '45" latitude sul e 34 $54^{\circ}$ '46" longitude oeste), região metropolitana de Recife (PE), no período de outubro a dezembro de 2002. Foram selecionadas e identificadas 13 hastes florais com inflorescências recém-emitas e facilmente visíveis em: variedades de Alpinia purpurata (touceiras com quatro a seis anos); cultivares de Anthurium andraeanum (plantas com um ano de transplantadas); Heliconia rostrata e $H$. rauliniana (touceiras com quatro anos); variedades de Etlingera elatior (touceiras com cinco anos); Zingiber spectabilis (touceiras com seis anos); Tapeinochilos ananassae (touceiras com dois anos); e Renanthera coccínea (plantas com dois anos).

Os parâmetros avaliados em relação às hastes florais foram: $\mathrm{CH}$ - comprimento da haste, mensurado da base da planta até a inflorescência de todos os genótipos; DH - diâmetro da haste, mensurado $50 \mathrm{~cm}$ abaixo da inflorescência para A. purpurata, E. elatior e T. ananassae e $20 \mathrm{~cm}$ para Z. spectabilis. Os parâmetros avaliados em relação às inflorescências foram: CI - comprimento da inflorescência de todos os genótipos; DI - maior diâmetro da inflorescência de A. purpurata, E. elatior, T. ananassae e Z. spectabilis. Foram utilizados paquímetro digital e trena para as medições.

Foi desenvolvida uma escala de notas para descrição do estádio de desenvolvimento das hastes florais: A. purpurata variedades Vermelha, Jungle Queen e Jungle King: a - emissão da inflorescência; b - inflorescência totalmente emitida; c - inflorescência abrindo; d - inflorescência com $2 / 3$ das brácteas abertas (ponto de corte); e - inflorescência com mais de $2 / 3$ das brácteas abertas (figura 1); A. andraeanum cultivares Eidibel, Cananéia e Champion: a - espata fechada visível sobre as folhas; $b$ - menos da metade da espata aberta; c - mais da metade da espata aberta; $\mathrm{d}$ - espata aberta com menos de 2/3 da espádice maduro; e $-2 / 3$ da espádice maduro (ponto de corte); $\mathrm{f}$ - mais de $2 / 3$ da espádice maduro (figura 2 ); $H$. rostrata e $H$. rauliniana: a - emissão da inflorescência; $b$ - inflorescência com uma bráctea aberta; c - inflorescência com duas brácteas abertas; d - inflorescência com três brácteas abertas; E. elatior variedades Vermelha e Porcelana: a - emissão da haste floral; b - formação da inflorescência; c - inflorescência abrindo; d - brácteas inferiores semi-abertas, parte central fechada (ponto de corte); e - brácteas inferiores abertas, parte central semi-aberta (ponto de corte); f - abertura total (figura 3); $Z$. spectabilis: a - emissão da inflorescência; b - inflorescência com diâmetro maior que o comprimento; $\mathrm{c}$ - inflorescência com comprimento igual ao diâmetro; $\mathrm{d}$ - inflorescência com comprimento maior que o diâmetro (ponto de corte); e - inflorescência extra grande (figura 4); T. ananasseae: a - emissão da inflorescência; b - inflorescência com comprimento igual ao diâmetro; c - inflorescência com comprimento maior que o diâmetro (ponto de corte); $R$. coccinea: a - surgimento da gema da haste floral (figura 5); b - ramificação da haste floral; $\mathrm{c}$ - haste floral com botões verdes; $d$ - haste floral com botões verdes e vermelhos; e - abertura das flores (ponto de corte).

As avaliações foram efetuadas em intervalos de sete a oito dias após a marcação (DAM) no início da emissão da inflorescência até a colheita. As análises de variância foram efetuadas utilizando o Programa SAS (SAS, 1999). Foram adotadas as transformações indicadas pelo procedimento Guided Analysis do mesmo programa, porém os dados são os originais. As médias dos tratamentos foram comparadas pelo teste de Tukey a $5 \%$ de probabilidade.

\section{RESULTADOS E DISCUSSÃO}

Foram observadas diferenças no desenvolvimento das hastes florais e das inflorescências entre as cultivares das espécies e durante o tempo avaliado (tabelas 1 a 5).

A largura e comprimento das inflorescências das variedades de A. purpurata aumentaram com o decorrer do tempo (tabela 1). Esse fato é esperado uma vez que ocorre a abertura da inflorescência. Já o comprimento e diâmetro da haste floral das variedades não alteraram ao 
longo das avaliações. Isto indica que, quando ocorre a emissão da inflorescência, as hastes florais já estão plenamente desenvolvidas não havendo um incremento do diâmetro ou comprimento. Portanto, hastes florais de A. purpurata que não tiverem a altura e o diâmetro desejados para corte, no início do florescimento, podem ser descartadas. Hastes florais fora do padrão comercial podem ser observadas em touceiras de diferentes idades, ou pode estar relacionado à falta de manutenção, a exemplo do excesso de perfilhos e deficiência de adubação.

Tomando-se como base o padrão descrito por LOGES et al. (2005b), uma haste floral de alpínia é considerada tipo A se a inflorescência apresentar $1 / 3$ das brácteas inferiores fechadas, diâmetro da haste maior que $10 \mathrm{~mm}$, além da ausência de danos. Como pode ser observado, em média, todas as flores atingiram este padrão (tabela 1).

Conforme a escala de notas adotada, as hastes florais de A. purpurata var. Vermelha poderiam ser colhidas a partir de 29 dias após a marcação (DAM), e as variedades Jungle King e Jungle Queen a partir de 37 e 43 DAM, respectivamente, quando as inflorescências ainda apresentam qualidade para corte.

Tabela 1. Desenvolvimento de hastes florais de variedades de Alpinia purpurata. Paulista, PE - 2002.

Tabela 1. Floral shoot emission of Alpinia purpurata varieties. Paulista, PE - 2002.

\begin{tabular}{|c|c|c|c|c|}
\hline \multirow[t]{2}{*}{$\begin{array}{l}\text { Parâmetros } \\
\text { Parameters }\end{array}$} & \multicolumn{4}{|c|}{$\begin{array}{c}\text { Dias após a marcação (DAM) } \\
\text { Days after shoot emition }\end{array}$} \\
\hline & 22 & 29 & 37 & 43 \\
\hline \multicolumn{5}{|l|}{ Nota/ Note * } \\
\hline Vermelha / Red & $2,63 \mathrm{aA} * *$ & $4,00 \mathrm{bA}$ & $4,00 \mathrm{bA}$ & $4,18 \mathrm{bA}$ \\
\hline Jungle Queen & $2,22 \mathrm{aA}$ & $3,75 \mathrm{bA}$ & $3,91 \mathrm{bA}$ & $3,91 \mathrm{bA}$ \\
\hline Jungle King & $2,20 \mathrm{aA}$ & $3,60 \mathrm{bA}$ & $4,10 \mathrm{bcA}$ & $4,40 \mathrm{cA}$ \\
\hline \multicolumn{5}{|c|}{ Comprimento inflorescência / Inflorescence length (cm) } \\
\hline Vermelha / Red & $15,65 \mathrm{bA}$ & $19,22 \mathrm{bA}$ & $24,45 \mathrm{aA}$ & $25,54 \mathrm{aA}$ \\
\hline Jungle Queen & $14,00 \mathrm{bA}$ & $16,83 \mathrm{bA}$ & $19,75 \mathrm{abA}$ & $21,75 \mathrm{aA}$ \\
\hline Jungle King & $19,50 \mathrm{aA}$ & $17,90 \mathrm{aA}$ & $21,20 \mathrm{aA}$ & $21,00 \mathrm{aA}$ \\
\hline \multicolumn{5}{|c|}{ Diâmetro inflorescência / Inflorescence diameter (mm) } \\
\hline Vermelha / Red & $37,09 \mathrm{bA}$ & $63,92 \mathrm{aA}$ & $66,18 \mathrm{aA}$ & $82,63 \mathrm{aA}$ \\
\hline Jungle Queen & $25,87 \mathrm{bA}$ & $54,34 \mathrm{aA}$ & $54,72 \mathrm{aA}$ & $64,88 \mathrm{aA}$ \\
\hline Jungle King & $40,50 \mathrm{bB}$ & $67,45 \mathrm{aB}$ & $75,88 \mathrm{aB}$ & $88,83 \mathrm{aB}$ \\
\hline \multicolumn{5}{|c|}{ Comprimento haste floral / Flower stem lenght (m) } \\
\hline Vermelha/Red & $1,31 \mathrm{aA}$ & $1,34 \mathrm{aA}$ & $1,32 \mathrm{aA}$ & $1,35 \mathrm{aA}$ \\
\hline Jungle Queen & $1,41 \mathrm{aA}$ & $1,47 \mathrm{aA}$ & $1,4 \mathrm{aA}$ & $1,42 \mathrm{aA}$ \\
\hline Jungle King & $2,44 \mathrm{aB}$ & $2,42 \mathrm{aB}$ & $2,50 \mathrm{aB}$ & $2,50 \mathrm{aB}$ \\
\hline \multicolumn{5}{|c|}{ Diâmetro haste floral / Flower stem diameter (mm) } \\
\hline Vermelha / Red & $13,35 \mathrm{aA}$ & $13,62 \mathrm{aA}$ & $14,08 \mathrm{aA}$ & $13,52 \mathrm{aA}$ \\
\hline Jungle Queen & $11,86 \mathrm{aA}$ & $13,29 \mathrm{aA}$ & $12,62 \mathrm{aA}$ & $13,19 \mathrm{aA}$ \\
\hline Jungle King & $18,47 \mathrm{aB}$ & $19,84 \mathrm{aB}$ & $20,25 \mathrm{aB}$ & $18,65 \mathrm{aB}$ \\
\hline
\end{tabular}

\footnotetext{
* Escala de notas descrita na metodologia

* Scale note described in metodology

** Médias na linha seguidas pela mesma letra minúscula e médias na coluna seguidas pela mesma letra maiúscula, não diferem entre si pelo Teste de Tukey $(\mathrm{P}<0,05)$.
} 
Observa-se que as $H$. rauliniana e $H$. rostrata atingiram esse ponto 15 DAM.

Os E. elatior variedades Vermelha e Porcelana atingiram o ponto de corte entre 36 e 42 DAM (tabela 4). Houve aumento do comprimento e diâmetro das inflorescências no decorrer do tempo como é esperado. Já o comprimento e diâmetro das hastes não diferiram entre 21 e 42 DAM. Isto indica que, após a formação da inflorescência não ocorre mais o aumento do comprimento e diâmetro da haste, como observado também nas variedades de A. purpurata. Portanto, hastes florais que não tenham comprimento e diâmetro adequados para a comercialização, até 21 dias após a emissão da inflorescência, podem ser removidas, pois não atingirão o padrão de qualidade. As variedades apresentaram o mesmo desenvolvimento nas épocas de avaliação. Segundo o Padrão de Comercialização de TEIXEIRA (2002), E. elatior tipo A, deve ter inflorescências com brácteas inferiores semi-abertas, parte central fechada e ausência de manchas.

O Z. spectabilis (tabela 5) atingiu o ponto de corte entre os 29 e 37 DAM sendo este mantido além dos 42 DAM, uma vez que podem ser colhidos com inflorescências maiores. O Padrão descrito por TEIXEIRA (2002) considera hastes tipo A, aquelas cujo comprimento das inflorescências são menores que $15 \mathrm{~cm}$, o caule está acima de $40 \mathrm{~cm}$ e as brácteas finais são fechadas.
Até os 42 DAM as hastes avaliadas encontravam-se nessa classificação.

As hastes florais de T. ananasseae (tabela 5) poderiam ser colhidas a partir de 29 DAM, pois apresentavam comprimento da haste acima de 1,47 $\mathrm{m}$ e comprimento da inflorescência maior que o diâmetro. São comercializadas tanto as inflorescências como os pseudocaules dessa espécie, considerada tipo A quando suas inflorescências e coloração são vermelho-intensa e comprimento do pseudocaule acima de 0,40 m (LOGES et al., 2005b).

As hastes florais da orquídea $R$. coccinea (tabela 5) atingiram o ponto de corte a partir dos 42 DAM. Foi observado que $17 \%$ das gemas marcadas não se transformaram em hastes florais. $\mathrm{O}$ comprimento da ramificação principal da haste floral colhida atingiu $53 \mathrm{~cm}$ aos 42 DAM

\section{CONCLUSÕES}

Por meio da caracterização do desenvolvimento de hastes de flores tropicais é possível prever o tempo necessário para colheita a partir da observação da data de emissão e das características do estádio de desenvolvimento da inflorescência.

Tabela 2. Notas atribuídas ao desenvolvimento de hastes florais de cultivares de Anturium andraeanum. Paulista, PE - 2002

Table 2. Notes atributed to A. andraeanum cultivars flower stems development. Paulista, PE-2002.

\begin{tabular}{|c|c|c|c|c|}
\hline \multirow[t]{2}{*}{$\begin{array}{r}\text { Cultivares } \\
\text { Cultivars }\end{array}$} & \multicolumn{4}{|c|}{$\begin{array}{l}\text { Dias após a marcação (DAM) } \\
\text { Days after shoot emission }\end{array}$} \\
\hline & 7 & 15 & 20 & 29 \\
\hline Eidibel & $1,08 * \mathrm{cA} * *$ & $1,42 \mathrm{cA}$ & $4,82 \mathrm{bA}$ & $5,75 \mathrm{aA}$ \\
\hline Cananéia & $1,42 \mathrm{cA}$ & $1,92 \mathrm{cA}$ & $4,73 \mathrm{bA}$ & $5,83 \mathrm{aA}$ \\
\hline Champion & $2,42 \mathrm{cB}$ & $3,58 \mathrm{bB}$ & $3,55 \mathrm{bB}$ & $6,00 \mathrm{aB}$ \\
\hline
\end{tabular}

\footnotetext{
* Escala de notas descrita na metodologia.

* Scale note described in metodology

** Médias na linha seguidas pela mesma letra minúscula e médias na coluna seguidas pela mesma letra maiúscula, não diferem entre si pelo Teste de Tukey $(\mathrm{P}<0,05)$.

** Means with the same letter are not statistically different according to Scott-Knott test, at 5\% probability.
}

Tabela 3. Desenvolvimento de hastes florais de Heliconia. Paulista, PE - 2002.

Table 3. Heliconia flower stems development. Paulista, PE - 2002.

\begin{tabular}{|c|c|c|}
\hline \multirow[t]{2}{*}{$\begin{array}{l}\text { Parâmetros } \\
\text { Parameters }\end{array}$} & \multicolumn{2}{|c|}{$\begin{array}{c}\text { Dias após a marcação (DAM) } \\
\text { Days after shoot emission }\end{array}$} \\
\hline & 9 & 15 \\
\hline \multicolumn{3}{|l|}{ Nota / Note* } \\
\hline H. rauliniana & $2,00 \mathrm{a}$ & $3,5 \mathrm{~b}$ \\
\hline H. rostrata & $2,25 \mathrm{a}$ & $4,18 b$ \\
\hline \multicolumn{3}{|c|}{ Comprimento inflorescência (cm) / Inflorescence length (cm) } \\
\hline H. rauliniana & $41,50 \mathrm{a}$ & $40,09 \mathrm{a}$ \\
\hline H. rostrata & $30,91 \mathrm{a}$ & $34,81 \mathrm{a}$ \\
\hline \multicolumn{3}{|c|}{ Comprimento haste floral (m) / Flower stem lenght (m) } \\
\hline H. rauliniana & $1,48 \mathrm{a}$ & $1,54 \mathrm{a}$ \\
\hline H. rostrata & $1,31 \mathrm{a}$ & $1,27 \mathrm{a}$ \\
\hline
\end{tabular}

\footnotetext{
* Escala de notas descrita na metodologia.

* Scale note described in metodology

** Médias na linha seguidas pela mesma letra, não diferem entre si pelo Teste de Tukey $(\mathrm{P}<0,05)$.

** Means with the same letter are not statistically different according to Scott-Knott test, at 5\% probability.
} 


\section{AGRADECIMENTOS}

Os autores aradecem à proprietária da Fazenda Mumbecas, Paulista (PE), local em que foram executadas as avaliações; ao Prof. Mario de Andrade Lira Junior (UFRPE) pelas orientações nas análises estatísticas; aos estagiários Paula G. L. Pinheiro, José Wendel de Oliveira Souza e Gustavo Jonnas S. M. Bezerra, pela colaboração na condução do experimento.

Tabela 4. Desenvolvimento de hastes florais de Etlingera elatior. Paulista, PE - 2002

Table 4. Etlingera elatior flower stems development. Paulista, PE-2002.

\begin{tabular}{|c|c|c|c|c|}
\hline \multirow[t]{2}{*}{$\begin{array}{l}\text { Parâmetro } \\
\text { Parameters }\end{array}$} & \multicolumn{4}{|c|}{$\begin{array}{c}\text { Dias após a marcação (DAM) } \\
\text { Days after shoot emission (DASE) }\end{array}$} \\
\hline & 21 & 27 & 36 & 42 \\
\hline \multicolumn{5}{|l|}{ Nota / Note * } \\
\hline Bastão Porcelana / Porcelain & $2,08 \mathrm{bA} * *$ & $2,82 \mathrm{bA}$ & $5,12 \mathrm{aA}$ & $5,33 \mathrm{aA}$ \\
\hline Bastão Vermelho / Red & $2,17 \mathrm{cA}$ & $3,36 \mathrm{bA}$ & $4,90 \mathrm{aA}$ & $5,67 \mathrm{aA}$ \\
\hline \multicolumn{5}{|c|}{ Comprimento inflorescência / Inflorescence length $(\mathrm{cm})$} \\
\hline Bastão Porcelana / Porcelain & $20,72 \mathrm{aA}$ & $16,90 \mathrm{aA}$ & $15,75 \mathrm{aA}$ & $17,66 \mathrm{aA}$ \\
\hline Bastão Vermelho / Red & $16,36 \mathrm{bA}$ & $18,27 \mathrm{abA}$ & $22,8 \mathrm{aA}$ & $20,16 \mathrm{abA}$ \\
\hline \multicolumn{5}{|c|}{ Diâmetro inflorescência / Inflorescence diameter (mm) } \\
\hline Bastão Porcelana / Porcelain & $30,88 \mathrm{bA}$ & $40,70 \mathrm{bA}$ & $97,98 \mathrm{aA}$ & $155,30 \mathrm{aA}$ \\
\hline Bastão Vermelho / Red & $34,02 \mathrm{bA}$ & $39,27 \mathrm{bA}$ & $88,1 \mathrm{aA}$ & $112,90 \mathrm{aA}$ \\
\hline \multicolumn{5}{|c|}{ Comprimento haste floral / Flower stem lenght (m) } \\
\hline Bastão Porcelana / Porcelain & $1,60 \mathrm{aA}$ & $1,61 \mathrm{aA}$ & $1,65 \mathrm{aA}$ & $1,50 \mathrm{aA}$ \\
\hline Bastão Vermelho / Red & $1,45 \mathrm{aA}$ & $1,52 \mathrm{aA}$ & $1,50 \mathrm{aA}$ & $1,46 \mathrm{aA}$ \\
\hline \multicolumn{5}{|c|}{ Diâmetro haste floral / Flower stem diameter (mm) } \\
\hline Bastão Porcelana / Porcelain & $18,86 \mathrm{aA}$ & $18,36 \mathrm{aA}$ & $17,69 \mathrm{aA}$ & $17,53 \mathrm{aA}$ \\
\hline Bastão Vermelho / Red & $18,02 \mathrm{aA}$ & $16,68 \mathrm{aA}$ & $17,68 \mathrm{aA}$ & $16,56 \mathrm{aA}$ \\
\hline
\end{tabular}

Tabela 5. Desenvolvimento de inflorescências de Zingiber spectabilis (sorvetão), Tapeinochilos ananasseae e Renanthera coccínea. Paulista, PE - 2002.

Table 5. Zingiber spectabilis, Tapeinochilos ananasseae and Renanthera coccinea inflorescense development. Paulista, PE-2002.

\begin{tabular}{|c|c|c|c|c|c|c|}
\hline \multirow{3}{*}{ Espécie / Specie } & \multicolumn{6}{|c|}{ Parâmetros / Parameters*** } \\
\hline & \multirow{2}{*}{$\begin{array}{l}\text { DAM }^{*} \\
D A S E^{*}\end{array}$} & \multirow{2}{*}{$\begin{array}{l}\text { Nota*** } \\
\text { Note** }\end{array}$} & \multicolumn{2}{|c|}{ Inflorescência / Inflorescence } & \multicolumn{2}{|c|}{ Haste floral / Flower stem } \\
\hline & & & Comp / Lenght & Diâm/ Diameter & Comp / Lenght & Diâm/ Diameter \\
\hline \multirow[t]{5}{*}{ Z. spectabilis } & & & $(\mathrm{cm})$ & $(\mathrm{mm})$ & $(m)$ & $(\mathrm{mm})$ \\
\hline & 22 & $2,00^{\mathrm{a}}$ & $4,94 \mathrm{a}$ & $48,94 \mathrm{a}$ & $51,45 \mathrm{a}$ & $15,57 \mathrm{a}$ \\
\hline & 29 & $2,89 \mathrm{~b}$ & $9,34 \mathrm{~b}$ & $64,41 b$ & $55,56 \mathrm{a}$ & $15,88 \mathrm{a}$ \\
\hline & 37 & $5,00 \mathrm{c}$ & $12,88 \mathrm{c}$ & $71,33 b$ & $59,67 \mathrm{a}$ & $15,73 \mathrm{a}$ \\
\hline & 42 & $5,00 \mathrm{c}$ & $13,87 \mathrm{c}$ & $71,36 b$ & $56,37 \mathrm{a}$ & $16,00 \mathrm{a}$ \\
\hline \multicolumn{7}{|l|}{ T. ananasseae } \\
\hline & 6 & 1,00 & $7,92 \mathrm{a}$ & $43,25 \mathrm{a}$ & $1,34 \mathrm{a}$ & $19,12 \mathrm{a}$ \\
\hline & 15 & 2,00 & $14,45 \mathrm{c}$ & $63,88 \mathrm{bc}$ & $1,36 \mathrm{a}$ & $19,24 \mathrm{a}$ \\
\hline & 20 & 2,00 & $12,33 b$ & $69,8 b c$ & $1,38 \mathrm{a}$ & $19,26 \mathrm{a}$ \\
\hline & 29 & 3,00 & $15,10 \mathrm{c}$ & $86,01 \mathrm{c}$ & $1,47 \mathrm{a}$ & $19,41 \mathrm{a}$ \\
\hline Espécie / Species & $\begin{array}{l}\mathrm{DAM} \\
\text { DASE* }\end{array}$ & $\begin{array}{l}\text { Nota* } \\
\text { Note** }\end{array}$ & \multicolumn{2}{|c|}{$\begin{array}{l}\text { Comprimento ramificação principal } \\
\text { Main dranch length }\end{array}$} & \multicolumn{2}{|c|}{$\begin{array}{l}\text { Comprimento haste } \\
\text { Stem lenght }\end{array}$} \\
\hline$R$. coccinea & & & \multicolumn{2}{|c|}{$(\mathrm{cm})$} & \multicolumn{2}{|c|}{$(\mathrm{m})$} \\
\hline & 22 & $2,42 \mathrm{a}$ & \multicolumn{2}{|c|}{$22 \mathrm{a}$} & \multicolumn{2}{|c|}{$1,61 \mathrm{a}$} \\
\hline & 28 & $2,83 \mathrm{a}$ & \multicolumn{2}{|c|}{$33 b$} & \multicolumn{2}{|c|}{$1,85 \mathrm{a}$} \\
\hline & 37 & $3,25 \mathrm{ab}$ & \multicolumn{2}{|c|}{$47 \mathrm{c}$} & \multicolumn{2}{|c|}{$1,82 \mathrm{a}$} \\
\hline & 42 & $4,00 \mathrm{~b}$ & \multicolumn{2}{|c|}{$53 \mathrm{~cd}$} & \multicolumn{2}{|c|}{$1,80 \mathrm{a}$} \\
\hline & 51 & $3,40 \mathrm{~b}$ & \multicolumn{2}{|c|}{$61 d$} & \multicolumn{2}{|c|}{$1,74 \mathrm{a}$} \\
\hline
\end{tabular}

\footnotetext{
* DAM - dias após a marcação;

* DASE - After days emission shoots;

** Escala de notas descrita na metodologia;

** Scale note described in methodology;

***Médias na linha seguidas pela mesma letra minúscula e médias na coluna seguidas pela mesma letra maiúscula, não diferem entre si pelo Teste de Tukey $(\mathrm{P}<0,05)$.
} 


\section{REFERÊNCIAS}

CASTRO, C.E.F. Helicônias como flores de corte: adequação de espécies e tecnologia pós-colheita. Piracicaba: Escola Superior de Agricultura Luiz de Queiroz, 1993. 191p. ilus.Tese de Doutorado.

JUNQUEIRA, A.H.; PEETZ, M.S. Las exportaciones brasileñas de flores y plantas crecen más del $124 \%$ entre 2001 y 2006. Revista Horticultura Internacional. v.56, 76$78,2007$.

LOGES, V.; CASTRO, A.C.R. de; MARTINS, L.S.S.; TEXEIRA, M. do C.F. Curso prático de pós-colheita para WILLADINO, L.G.; TEXEIRA, M.C.F.; LIRA JUNIOR, flores tropicais. In: SEBRAE. Floricultura em Pernambuco. M.A.; PINHEIRO, P.G.L.; SOUZA, J.W.O; MELO, M.G. Recife: SEBRAE/PE (Série Agronegócio). 11-16. 2002.
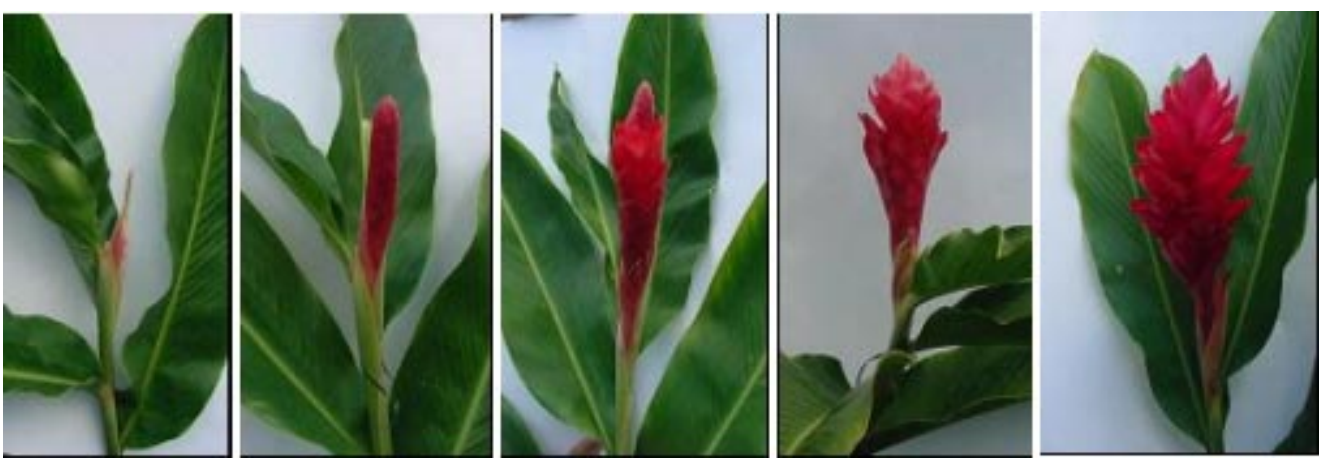

Figura 1. Escala de notas para descrição do estádio de desenvolvimento das hastes florais de A. purpurata: a - emissão da inflorescência; b - inflorescência totalmente emitida; c - inflorescência abrindo; $d$ - inflorescência com $2 / 3$ das brácteas abertas (ponto de corte); e - inflorescência com mais de 2/3 das brácteas abertas.

Figure 1. Scale note describing flower stems development of A. purpurata varieties: a - inflorescence emission; $b-$ inflorescence fully emited; $c$ - inflorescence oppening; $d$-inflorescence with 2/3 oppened bracts (harvest point); $e-$ inflorescence with more than 2/3 oppened bracts.
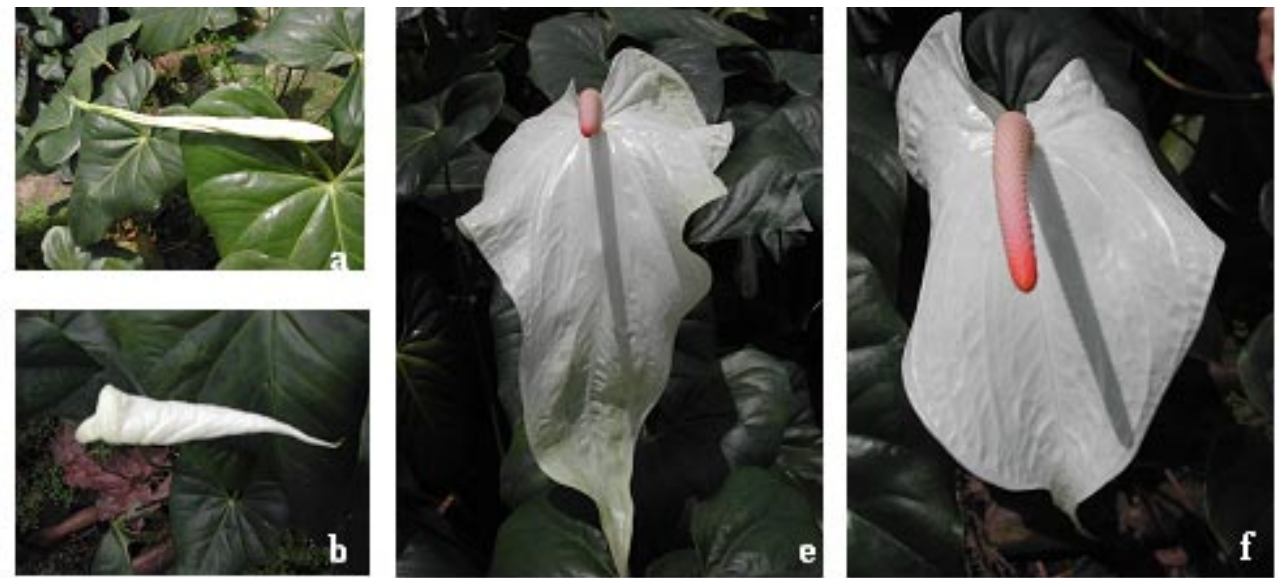

Figura 2. Escala de notas para descrição do estádio de desenvolvimento das hastes florais de A. andraeanum: a - espata fechada visível sobre as folhas; $b$ - menos da metade da espata aberta; e - 2/3 da espádice maduro (ponto de corte); f mais de 2/3 da espádice maduro.

Figure 2. Scale note describing flower stems development of A. andraeanum: a - closed (bracts) visible above the leaves; $b$-bracts with less than half the way opened; $e$-inflorescence $2 / 3$ madure (harvest point); $f$-showing more than 2/3 madure. 

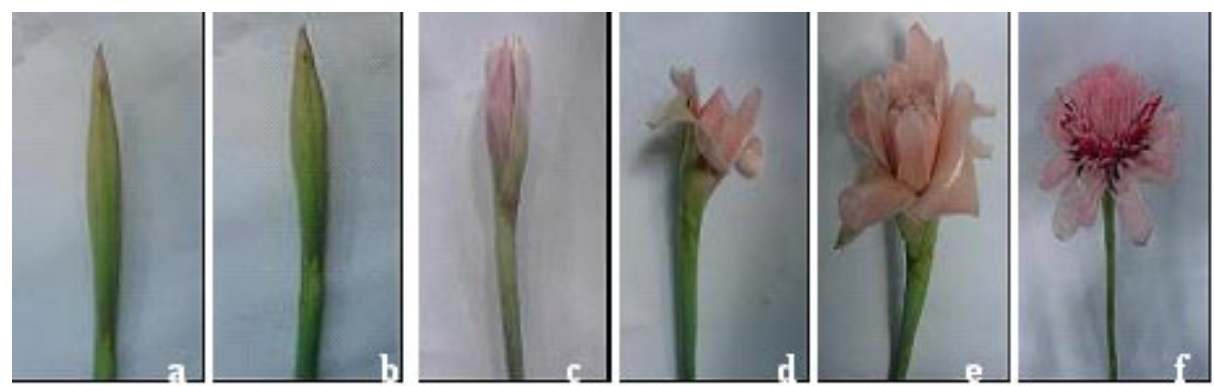

Figura 3. Escala de notas para descrição do estádio de desenvolvimento das hastes florais de E. elatior: a - emissão da haste floral; b - formação da inflorescência; c - inflorescência abrindo; d - brácteas inferiores semi-abertas, parte central fechada (ponto de corte); e - brácteas inferiores abertas, parte central semi-aberta (ponto de corte); f - abertura total.

Figure 3. Scale note describing E. elatior flower stems development stages: a -flower stem emission; $b$ - formation of the inflorescence; $c$ - opening inflorescence; $d$ - under bracts partially opened and central portion closed (harvest point); $e$ - under bracts opened and central portion partially opened (harvest point); $f$ - tottaly opened.
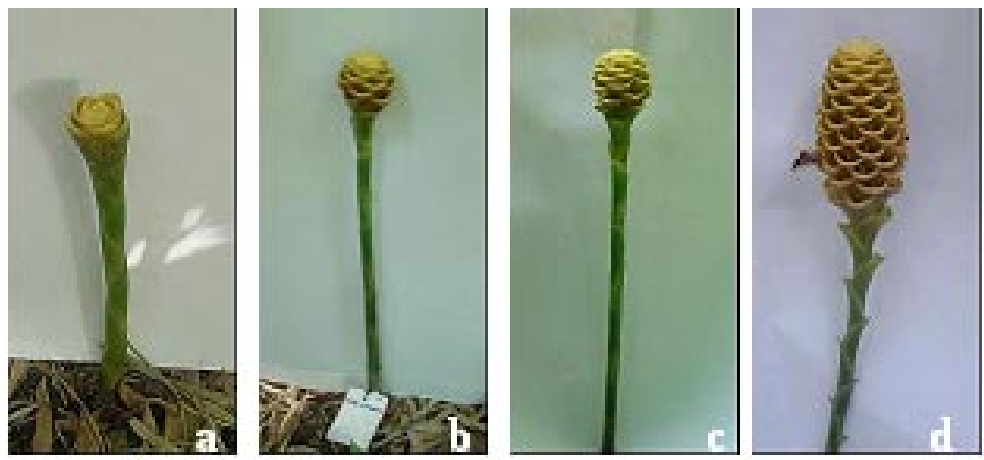

Figura 4. Escala de notas para descrição do estádio de desenvolvimento das hastes florais de Z. spectabilis: a - emissão da inflorescência; $b$ - inflorescência com diâmetro maior que o comprimento; c - inflorescência com comprimento igual ao diâmetro; $\mathrm{d}$ - inflorescência com comprimento maior que o diâmetro (ponto de corte).

Figure 4. Scale note describing $\underline{Z}$. spectabilis flower stems development stages: $a$ - inflorescence emission; $b-$ inflorescence with diameter greater than length; $c$ - inflorescence with length equal to diameter; $d$ - inflorescence with length than diameter (harvest point).
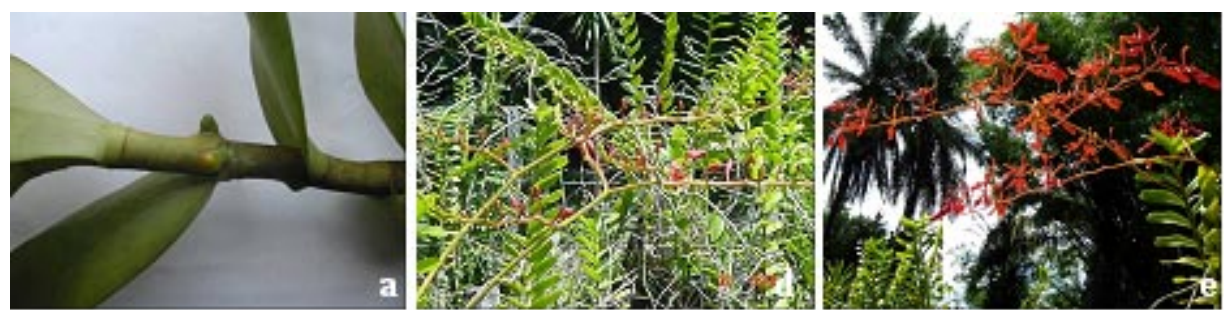

Figura 5. Escala de notas para descrição do estádio de desenvolvimento das hastes florais de $R$. coccinea: a - surgimento da gema da haste floral; $\mathrm{d}$ - haste floral com botões verdes e vermelhos; e - abertura das flores (ponto de corte).

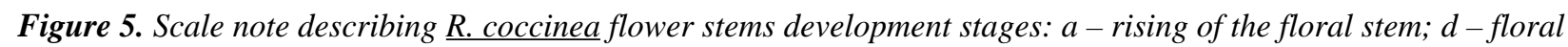
stem abowing green and red; $e$-flower blooming (harvest point). 